

Weidner, Verena (2007): »Interpretation« - VII. Jahreskongress der Gesellschaft für Musiktheorie und 6th European Music Analysis Conference, Freiburg 11. bis 14. Oktober 2007. ZGMTH 4/3, 333-343. https://doi.org/10.31751/266

(C) 2007 Verena Weidner

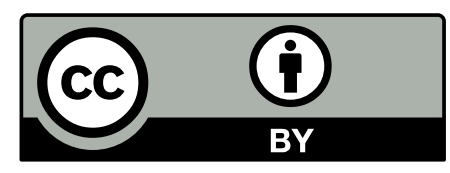

Dieser Text erscheint im Open Access und ist lizenziert unter einer Creative Commons Namensnennung 4.0 International Lizenz.

This is an open access article licensed under a Creative Commons Attribution 4.0 International License.

veröffentlicht / first published: 01/07/2007

zuletzt geändert / last updated: 04/07/2009 


\section{»Interpretation« - VII. Jahreskongress der Gesellschaft für Musiktheorie und 6th European Music Analysis Conference, Freiburg 11. bis 14. Oktober 2007}

`Viek « und `Vielfältig ` - das sind Stichworte, wie sie einem zu der internationalen Tagung in den Sinn kommen, die unter dem Thema >Interpretation vergangenen Jahres in Freiburg im Breisgau stattfand. Allein mit Programm und Abstracts hält man ein mehr als hundert Seiten starkes Heft in Händen. An die 180 Beiträge in zahlreichen Sektionen, Projekt-Präsentationen, Workshops und Kolloquien sowie ein Rahmenprogramm mit Konzerten und Führungen boten den über 250 Kongressteilnehmern eine unglaubliche Fülle an Anregungen und Eindrücken.

Möglich wurde das voluminöse Unternehmen durch die Konzeption als Doppelkongress: Der VII. Jahreskongress der Gesellschaft für Musiktheorie wurde erweitert um die 6th European Music Analysis Conference, die nach den Vorläufer-Konferenzen in Frankreich, Italien, den Niederlanden und England damit erstmalig in Deutschland stattfand. Lange war auf dieses Ziel hingearbeitet worden, und Beträchtliches hatte man sich vorgenommen. So hieß es im Call for Papers: »In der Tradition der europäischen Kongresse für musikalische Analyse stehend, ist es den Veranstaltern ein besonderes Anliegen, die unterschiedlichen nationalen Forschungstraditionen zusammenzuführen."Als Veranstalter firmierten die Musikhochschule Freiburg, die Gesellschaft für Musiktheorie, die Gesellschaft für Musik und Ästhetik und die Albert-Ludwigs-Universität Freiburg, die den Kongress zu einem zentralen Programmpunkt ihrer 550-Jahrfeier gemacht hatte. Die Kooperation zwischen den beteiligten Organisationen und Institutionen war denn auch ein thematischer Angelpunkt der Begrüßungen durch Dr. Karl-Reinhard Volz (Prorektor der Albert-Ludwigs-Universität Freiburg), Dr. Rüdiger Nolte (Rektor der Musikhochschule Freiburg), Stefan Rohringer (Präsident der GMTH) und Ludwig Holtmeier (Konferenzleitung).

Gemäß der Internationalität des Publikums waren als Konferenzsprachen Deutsch, Englisch und Französisch zu hören - besonders eloquent miteinander verknüpft in der Begrüßungsrede Ludwig Holtmeiers, der nach seiner Federführung beim Gründungskongress der Gesellschaft für Musiktheorie in Dresden 2001 mit der Freiburger Veranstaltung bereits sein zweites Meisterstück ablieferte. Nicht minder hervorzuheben ist die Arbeit der übrigen Mitglieder der Kongressleitung, Dr. Günter Schnitzler für das `Studium Generale` und Florian Vogt für das Musikwissenschaftliche Seminar, beide Albert-Ludwigs-Universität, sowie Dr. Janina Klassen und Dr. Johannes Menke für die Hochschule für Musik Freiburg, nicht zu vergessen die zahlreichen namenlosen Helfer, die für einen reibungslosen organisatorischen Ablauf und eine wohltuend entspannte Atmosphäre sorgten.

Das reichhaltige Angebot machte es erforderlich, dass bis zu vier Sektionen parallel stattfanden. Die Berichterstattung durch eine Einzelperson kann daher nur den Versuch bedeuten, zwischen der notwendigerweise subjektiven Beitragsauswahl und einem angemessenen Überblick über die Fülle der Themen zu vermitteln. 


\section{Konzeption}

Wie bei den Kongressen der Vorjahre ließ der Call for Papers eine thematische Gliederung in mehrere Hauptsektionen erwarten. Angekündigt waren neben freien Beiträgen die Sektionen »Musikalische Analyse und Aufführungspraxis«, »Text und Musik«, »Zur Interpretation Neuer Musik«, »Zum Begriff der Improvisation im 18. Jahrhundert« und »Formenlehre«. Am Ende ermöglichte die Menge und Vielfalt der eingegangenen Abstracts jedoch neue und unvorhergesehene thematische Bündelungen und machte die Trennung zwischen thematischen und freien Sektionen hinfällig. Nicht immer profitierte davon die Ausdifferenzierung des eher unspezifisch gehaltenen Kongressthemas, bisweilen litt auch die Kontur der einen oder anderen Sektion. Um etwaigen Fliehkräften entgegenzuwirken, konzipierte man einzelne Sektionen als in sich geschlossene Konferenzen. So führte beispielsweise Pieter Bergé (Leuven) ein eigenständiges Panel innerhalb der Sektion »Formenlehre« durch. In den regulären Programmablauf eingebunden waren ferner das von Richard Klein (Freiburg) geleitete Symposium »Stimme« sowie das Symposium "Zahl und Struktur in Josquins Werk», dem Christian Berger (Freiburg) vorstand.

Einen ersten fachlichen Einstieg bot der Eröffnungsvortrag, gehalten von Eckehard Kiem (Freiburg). Kiem gab am Beispiel Jacobus Vaets einen Einblick in Methoden der Analyse Alter Musik, die sich nicht auf bloße Generalisierungen beschränken, sondern gerade das Individuelle und Werkhafte der Stücke sichtbar machen.

\section{Musikalische Analyse}

\section{Formenlehre}

Zu den Kernsektionen des Kongresses zählte als «klassische` musiktheoretische Disziplin die »Formenlehre«. In dieser Sektion kamen grundlegende systematische Erörterungen neben der Betrachtung spezifischer Modelle und historischer Ausprägungen zu stehen. So beleuchtete Florian Edler (Berlin) die »systematische und stilgeschichtliche Problematik von Grundbegriffen der Deutschen Formenlehre» an den Beispielen 'Satzı, >Barform und `Fortspinnungstypus`, und Emil Platen (Bonn) thematisierte den `Dreischritt »elementares Formprinzip der tonalen Musik«. Eine Differenzierung der »syntaktischen Typen >Periode` und `Satz« geschah durch Stefan Rohringers (München) Rekurs auf die Unterscheidung zwischen sStrukturı und sDesign`. Hiervon ausgehend fragte er nach der wechselseitigen Übersetzbarkeit der Theorien Schenkers und Schönbergs. Patrick McCreless (Yale) untersuchte aufführungsspezifische Implikationen der für Schönberg und Ratz zentralen Begriffe `Auflösung` und `Verkürzung`. Thomas Noll (Berlin) zielte beim Thema Fundamentalbassfortschreitungen auf eine Integration schenkerianischer und rameauscher Prinzipien. Christian Raff (Stuttgart) und Wolfgang Winterhager (Bochum) wandten sich mit den Themen »Tempo di Menuetto als Satzüberschrift» und »Walzer im Variationenzyklus« jeweils gattungstheoretischen Fragen zu. Johannes Kreidler (Detmold) schließlich erörterte die Bedeutung des Formbegriffs Niklas Luhmanns für die Musiktheorie. Um eher rezeptionsorientierte Kategorien ging es Martin Küster 
(Ithaca/NY), der die Frage nach einem musikalischen `Urmeter am Beispiel von `Gavotte` und `Polonaise` zu klären versuchte. Auch Ulrich Kaiser (München) legte besonderen Wert auf die Frage der `Auffassung`: Anhand der Struktur von modulierenden und nichtmodulierenden Überleitungen in Mozarts früher Klaviermusik stellte er der gängigen Modulationslehre eine historische Perspektive gegenüber. Weitere Vorträge bezogen sich jeweils auf einen bestimmten Musikstil. So betrachtete Michel Vallières (McGill) die Kadenzvorbereitung in Corellis Violinsonaten op. 5. Einer sehr konkreten Untersuchung der musikalischen Syntax unterzog Markus Neuwirth (Leuven) die Reprisen in Joseph Haydns Klaviersonaten.

Drei Vorträge befassten sich mit der Musik Franz Schuberts, deren konstruktive Formgestaltung zunehmend zum Forschungsgegenstand wird. Anne Margaret Hyland (Cambridge/UK) versuchte in Auseinandersetzung mit Schuberts formalen Strategien den Begriff der >Teleologie` neu zu fassen. Dabei legte sie Wert auf eine Trennung zwischen grammatischem und rhetorischem `Ziek und betonte, das rhetorische Telos ginge über die von der Sonatentheorie beschriebene Grammatik hinaus. Anders gelagert war die Beschäftigung Laura Krämers (Berlin) mit »Form und Soziolekt« in Schuberts Tänzen, deren individuelle Art der Formgestaltung Thomas Fesefeldt (Hannover) betonte.

Ein eigener Sektionsabschnitt war dem 19. Jahrhundert gewidmet. Hier war insbesondere der Vortrag Janet Schmalfeldts (McGill) mit Spannung erwartet worden. Schmalfeldts Blick auf die `Ascending-Thirds Progression` bei Chopin zeigte nicht nur die große Prominenz dieses Fortschreitungsmodells in dessen Kompositionen, sondern deckte implizit auch die terminologischen und systematischen Unterschiede auf, die in der Beschreibung musikalischer Topoi zwischen der amerikanischen und insbesondere der deutschen Tradition herrschen. Eher übergeordneten Gesichtspunkten war das Referat »Bestimmbarkeit der Form bei Richard Wagner« von Ariane Jeßulat (Würzburg) verpflichtet. Jeßulat setzte sich kritisch mit dem in der Wagnerforschung vorherrschenden Problem der Verbindung von Detail- und großformaler Analyse auseinander und versuchte, am Finale der Walküre einen integrativen Ansatz zu entwickeln.

Auch Formbildungen in der Musik des 20. Jahrhunderts wurden diskutiert. So verglich Dániel Péter Biró (Victoria/CA) Gustav Mahlers `Neunte und Anton Weberns Sinfonie op. 21. Pascal Decroupet (Antipolis) mit seinen Überlegungen zu einem realzeitlichen Formbegriff und Stephen Downes (Surrey/UK) mit dem Thema »Musikalische Form und Dekadenz« nahmen schließlich explizit Formbildung in der Neuen Musik in den Blick.

\section{Methodologie}

Einen weiteren Schwerpunkt, mit thematischen Schnittmengen zur Sektion »Formenlehre», bildete die Sektion »Methodologie«: Auch hier wurden sowohl systematische Überlegungen bis hin zur wissenschaftstheoretischen Reflexion angestellt, als auch spezifische Musikstile bzw. Werke einzelner Komponisten thematisiert.

Recht speziell war das Thema von Jean-Marc Chouvel (Paris), der sich der Anwendung des Fourier-Differentials auf die Interpretationsanalyse widmete. Als in ihrer Reichweite historisch begrenzt, für die Praxis jedoch bis heute attraktiv, erwies sich die von Christhard Zimpel (Weimar) untersuchte `Tonika-Do-Methode` von Maria Leo. Jan-Phi- 
lipp Sprick (Rostock/Berlin) erforschte auf Basis von bisher unveröffentlichtem Quellenmaterial die Aufführungstheorie Rudolf Kolischs. Dabei legte er besonderes Augenmerk auf die Frage nach deren Aktualität in Zeiten der shistorischen Aufführungspraxis‘. Auf thematisch verwandtem Gebiet bewegte sich Thomas Kabisch (Trossingen), der August Halms Ideen zur Aufführungspraxis vorstellte.

Fragen der ästhetischen Wahrnehmung leiteten Daniel Shanahan (Dublin), der mit Bezug auf die Husserlsche Phänomenologie das Moment der »Melodischen Erwartung" untersuchte. Unter ähnlicher Prämisse beschäftigte sich das italienische Trio, bestehend aus Rossana Dalmonte (Trento), Mario Baroni (Trento) und Roberto Caterina (Bologna), mit der Wahrnehmung melodischer Konturen, während Mario Videira (Tübingen) den Zusammenhang von »Musik und Subjektivität in der deutschen klassischen Philosophie» nachzeichnete.

Nikolaus Schneider (Bonn) und Jakob Ullmann (Basel) widmeten sich der Frage der Sprachähnlichkeit von Musik. Schneider übte sich hierbei in der »Kritik eines erfolgreichen Paradigmas« während Ullmann auf die Interdependenz zwischen »Buchstaben und Musik« in der Tradition griechisch-antiker und mittelalterlicher Musik aufmerksam machte.

Eng am Kongressthema ausgerichtet war der Vortrag von Michel Imberty (Paris) und Aurélie Fraboulet (Paris), die den Begriff des ıInterpretierens« als »Erzählen einer Geschichte« metaphorisierten. In eine ähnliche Richtung wies Beate Perrey (Liverpool) mit der Betonung des performativen und künstlerischen Aspekts von Analyse. Auf einzelne Werke bezogen sich hingegen Ildar Khannanovs (Santa Barbara) Untersuchung von Tchaikovskys Romeo und Julia sowie das Referat von Gerhard Lock (Tallin), der den formalen Spannungsverlauf in einzelnen Sinfonien Schostakovitschs nachzeichnete.

Auch in dieser Sektion lag ein besonderer Schwerpunkt auf der Beschäftigung mit zeitgenössischer Musik. So machte José Oliveira Martins (lowa) auf die teils frappante Nähe zwischen musikalischen Konzeptionen des Mittelalters und der Moderne aufmerksam. Yulia Kreinin (Jerusalem) erkannte in Paul Klees »Bewegungsformen [...] ein Instrument der Analyse von Musik des 20. Jahrhunderts«. Robert Zierolf (Cincinnati) stellte Analyseansätze für die Musik des Futurismus vor, während Michael Clarke (Huddersfield) einen »interaktiv hörenden Zugang« für elektroakustische Musik generierte.

\section{Metapher}

Im Kontext der Methodologie wurde nicht zuletzt die sMetapherı als Grundprinzip musiktheoretischen Sprechens erörtert. Einen umfassenden Überblick über die musikanalytischen Perspektiven dieses originär sprachwissenschaftlichen Begriffs gaben Christian Thorau (Frankfurt/Main) und Robert Hatten (Indiana). Thorau griff dabei vor allem neuere Ansätze auf, die eine nichtsprachliche und multimodale Metaphorik im Blick haben und sich deshalb besonders für einen Transfer in die Musikanalytik eignen. Auf Paul Ricoeur und die hermeneutische Tradition rekurrierte Michael Spitzer (Durham), der sich hiervon Neuerungen für die Sonatentheorie erhoffte. Martin Kaltenecker (Paris) wiederum charakterisierte die »Metaphorisierung von Musik als musikalische Zuschreibung", in der sich Versprachlichung und Verbildlichung auf besondere Weise verbinden. Abgerundet wurde die Sektion durch die lebendige Darstellung Janina Klassens (Freiburg), 
die am Beispiel von Kommentaren Hanslicks zum Klaviervortrag Clara Schumanns die Interpretationsbedürftigkeit von Tempobezeichnungen aufzeigte.

\section{Theorien und Methoden}

\section{Historische Satzlehre}

Auch der Freiburger Kongress zeugte von dem nach wie vor ungebrochenen Trend zur Historischen Satzlehre. Die Vorträge von Angelika Moths (Basel/Bremen), Luca Bruno (Dallas/TX) und Antonio Cascelli (Hull/UK) ließen dies am Beispiel der Analyse von Renaissance-Musik deutlich werden. Dem klassisch-romantischen Bereich widmeten sich Felix Diergarten (Basel), der die Kompositionslehre Johann Friedrich Daubes vorstellte, und Folker Froebe (Mannheim/Hannover), der mit Hilfe systematischer Rekomposition die »Neuinszenierung traditioneller Modelle in Robert Schumanns Klaviermusik« offenlegte.

Birger Petersen (Rostock/Greifswald) zeigte Möglichkeiten, die »Orgelchoralsätze der Neumeister-Sammlung im Tonsatzunterricht« zu verwenden. Michael Malkiewicz (Salzburg) und Hubert Moßburger (Bremen) thematisierten das Verhältnis von Analyse und Aufführungspraxis und Ugo Piovano (Turin) nahm »L'Art de Preluder von Hotteterre» zum Ausgangspunkt, um auf das hohe Maß an (musiktheoretisch fundierter) >Regelsteuerung in der »Improvisationspraxis im 18. Jahrhundert« hinzuweisen.

Durch einen deutlichen Praxisbezug waren auch die Beiträge zur Partimento-Tradition geprägt. Die Wiederentdeckung dieser bedeutenden Lehrtradition ist spätestens seit der Eastern Orpheus Academy 2006 in aller Munde. Robert O. Gjerdingen (Northwestern), der zahlreiche Partimento-Quellen erschlossen hat, gab einen Überblick über neapolitanische Solfeggi als Modelle für die Improvisation. Gaetano Stella (Rom) verwies am Beispiel von Pietro Raimondi und Pietro Platania auf Zusammenhänge mit der Kontrapunktlehre des 19. Jahrhunderts. Ludwig Holtmeier (Freiburg/Basel) versuchte den »Tonalitätsbegriff« der Partimento-Tradition in Abgrenzung von jenem der ramistischen Lehre zu spezifizieren und betonte, die hinter dem Partimento stehende theoretische Konzeption gehe über den gemeinhin mit der Generalbasspraxis verbundenen Handwerksbegriff deutlich hinaus.

\section{Schenkerian Studies und Set Theory}

Auch im deutschsprachigen Raum haben sich die 'Schenkerian-Studies` mittlerweile etabliert. Neben konkreten Analysen, etwa zur "Stimmführung in Ravels G-Dur-Konzert« von Xavier Hascher (Strasbourg) oder zur »Tonalität und Form in Mozarts Opernfinales« von William Marvin (Rochester/NY) wurde auch die Schenker-Tradition selbst aufgearbeitet. So stellte Christoph Hust (Mainz) »Neue Quellen zu Heinrich Schenkers Vortragslehre« vor, und John Koslovsky (Rochester/NY) widmete sich den bislang unveröffentlichten »Schriften Felix Salzers an der New York Public Library«. Don Traut unterzog Schenkers Paradigmen zum stiefen Mittelgrund einer Reflexion und Andreas 
Moraitis (Berlin) evaluierte dessen späte Analytik. Interdisziplinäre Aspekte beleuchtete Hans-Ulrich Kretschmer (Zürich) in "Schenker-Analyse als Mittel zur Kritik musikalischer Aufführungspraxis« sowie Bertil Wikman (Stockholm), der auf Parallelen zwischen dem musikalischen Denken Heinrich Schenkers und dem Artur Schnabels hinwies - was vor dem Hintergrund der von Hust zuvor dargelegten Antipathie Schenkers gegenüber Schnabels Klavierspiel nicht ohne Pikanterie war.

Das Streben nach integrativen Ansätzen war auch bei den Beiträgen zur Sektion "Set Theory« zu spüren. So fragte Timothy L. Jackson (North Texas), ausgehend von unveröffentlichten Analysen Allen Fortes, nach Möglichkeiten, lineare und settheoretische Zugänge zu verbinden. Stephan Lewandowski (Dresden) brachte den Tonalitätsbegriff bei Roy Travis ins Spiel, um einen `Schenkerian approach` für die `Klassische Moderne` aufzuzeigen. Michel Schuijer (Amsterdam) schließlich diskutierte den ontologischen Status der »Pitch-Class Set Inclusion«.

\section{Musik und Sprache}

\section{Kunstlied}

Als einziger Gattung war dem Kunstlied eine eigene Sektion gewidmet. Den Anfang machte Harald Krebs (Victoria/CA) mit einem Referat über die »Inkongruenz von Text und Musik in den späten Liedern von Robert Schumann«. Dabei fokussierte er besonders einen Liedtypus, bei dem das regelmäßige musikalische Hypermeter und der ebenfalls metrisch regelmäßig gegliederte Text nicht kongruieren. »Wissenschaftstheoretische Probleme der Liedanalyse« thematisierte Arno Lücker (Berlin), der den Ansatz Kofi Agawus kritisch prüfte und dessen Ergebnisse mit jenen anderer Analyseverfahren verglich. Das moderne Kunstlied schließlich fand mit Frederik Knops (Hamburg) Überlegungen zu »Vokalstimme und Instrumenten in György Ligetis letzten Liedern« sowie Robert Cooks (lowa) Vortrag zu Liedern von George Crumb Beachtung.

\section{Musik und Text}

Ergänzt wurde die Sektion »Kunstlied« durch die sich anschließende Vortragsreihe zu »Musik und Text«. Annick Dubois (Frankreich) zeigte, wie Marc Antoine Charpentier die Rede vom Tod in musikalische Poetik übertrug. Luigi Collarile (Freiburg/Basel) verwies auf das wechselseitige Abhängigkeitsverhältnis zwischen der Erneuerung der Motette im 17. Jahrhundert und der Entstehung einer neuen, an die zeitgenössische italienische Lyrik anknüpfenden »lateinischen Dichtung für Musik«. Die Korrelation zwischen den zueinander querstehenden Symmetrien des textlichen und des tonalen Aufbaus in Bachs Actus tragicus nahm Toomas Siitan (Tallin) in den Blick.

Daneben spielte die Oper eine wichtige Rolle. So setzte Ana Stefanović (Belgrad) die Opern von Hector Berlioz in Beziehung zur französischen Barockoper, Andrew Davis (Houston) untersuchte die strukturelle Symmetrie in Hugo von Hofmannsthals Libretto Ariadne auf Naxos. Nicholas Baragwanath (Manchester) stellte am Beispiel von Giacomo 
Puccinis Tosca dar, wie sich in der Aufführungspraxis der italienischen Oper des späten 19. Jahrhunderts die Gestik der Ausführenden zugunsten einer mimetischen Expressivität von der Bindung an Leitmotivik und musikalische Struktur löste. Florian Wetter (Freiburg) schließlich analysierte die Funktion von Bela Bartóks Musik für Saiteninstrumente, Schlagzeug und Celesta als »filmischer Subtext« von Stanley Kubricks The Shining.

\section{Musikalische Moderne}

\section{Wiener Schule}

Mehrere Sektionen waren dem weiten Feld der musikalischen Moderne gewidmet. Allein elf Referate befassten sich mit Theorie und Musik der 'Wiener Schule`.

Die Theorie Schönbergs stand bei Christopher Wintle (London) und Norton Dudeque (Parana) im Zentrum. Wintle fokussierte hierbei die Begriffe `Entwicklung`, $A$ Abwicklung s und `Aneinanderreihung، in Schönbergs Formtheorie, woraufhin Dudeque die analytische Theorie Schönbergs zu systematisieren versuchte. Das »Verhältnis von Analyse, Formenlehre und Interpretation in der Wiener Schule« thematisierte Nikolaus Urbanek (Wien). Lubormír Spurný (Brno) ergänzte die Theoriereflexion um »einige Bemerkungen zu Alois Hábas Neuer Harmonielehre«. Hans-Niklas Kuhn (Luzern) nahm das Wort-Ton-Verhältnis der George-Lieder Schönbergs in den Blick. Walter Kreyszig (Saskatoon/CA) beschäftigte sich mit der Bedeutung des >Bach-Motivs` in Weberns Werk, Wally Yu (Austin) untersuchte das Verhältnis von »Tonalität, Atonalität und Intervall-Zyklen in Schönbergs Ein Stelldichein und dem Streichquartett Nr. 2« und Mauro Mastropasqua (Bologna) setzte "Set und Syntax in Schönbergs Suite op. 25« in Korrelation. Maria Sourtzi (Pallini) schloss die Sektion ab, indem sie »Symmetrieverhältnisse in Anton Weberns Symphonie op. 21« zum Vorschein brachte.

\section{Berliner Schule}

Mit Erich Schmid und Nikos Skalkotta fanden Kompositionsschüler Schönbergs Erwähnung, die heute der so genannten `Berliner Schule، zugerechnet werden. Skalkottas dodekaphone Werke wurden von Eva Mantzourani (Canterbury) auf »Kohärenz, Formenlehre und Sonaten-Deformation« hin untersucht, während Costas Tsougras (Thessaloniki) dessen Passacaglia besprach. Juliane Brandes (Freiburg) schließlich beschäftigte sich mit Schmids »Drei Sätzen für Orchester« op. 3, zu denen bis dato keine publizierte Analyse vorliegt. Sie machte besonders auf den individuellen Umgang mit den Kompositionsprinzipien der szweiten Wiener Schule` aufmerksam und stellte im Zuge der gerade beginnenden Renaissance der Werke Schmids weitere analytische Beiträge in Aussicht.

\section{Messiaen}

In der Sektion »Messiaen« referierte Siglind Bruhn (Michigan) zu »Bild, Text und Analyse im zehnten Lied aus Harawi: chant d'amour et de mort« während Wai Ling Cheong 
(Cambridge) Sept haïkaï für Klavier und kleines Orchester analysierte. Heather White Luckow (McGill) schließlich nahm das stransatlantische Erbe « Messiaens in den Blick.

\section{Neue Musik}

Den Beginn dieser Sektion machten Amanda Bayley (Wolverhampton) mit einer auf das Verhältnis von »Notation und Aufführung « abzielenden Analyse des 2. Streichquartetts von Michael Finnissy und Anne-Sylvie Barthel-Calvet (Metz), die mit der Beobachtung rhythmischer Konstellationen in Werken lannis Xenakis' ebenfalls Fragen der Aufführung in den Blick nahm. Auf ähnliche Weise wies später Sophie Dardeau (Paris) am Beispiel von Luciano Berios Sequenza I auf den Aspekt der Kreativität bei der Interpretation neuer Musik hin. Nathalie Ruget (Rouen) analysierte die letzten Werke Luigi Nonos im Hinblick auf ihre vokale und instrumentale Interpretation, und Paolo Dal Molin (Rouen) fand Zusammenhänge zwischen Pierre Boulez' Codomaines und seinem Werdegang als Solist.

Kompositionstheoretisch hingegen war das Interesse Janine Droeses (Hamburg), die anhand von Gérard Griseys Vortex temporum nach dem »Verhältnis der spektralen zur seriellen Musik« fragte. Auch Balz Trümpy (Basel) hatte in »Feldharmonik: Luciano Berios Antwort auf den Strukturalismus" vor allem die Theorie im Blick, ebenso Maarten Quanten (Leuven/Berlin), der »Stockhausens Klavierstück I als Antizipation auf [sic] die Neue Morphologie der musikalischen Zeit« interpretierte. Neue Formen der Zeitgestaltung thematisierte auch Jean-Pascal Chaigne (Nizza) in seinem Vortrag zur »Stille als Träger der musikalischen Dramaturgie« in Brian Ferneyhoughs Kurze Schatten II.

Das gemessen am Gegenstand vergleichsweise kurze Referat Paolo Zavagnas (Florenz) zu Salvatore Sciarrinos elektroakkustischer Oper Persio e Andromeda rekonstruierte die computertechnische und ästhetische Übersetzung des Librettos in ein Maximum an klanglicher Abstraktion. Jean-François Trubert (Aix-en-Provence) beschäftigte sich mit Mauricio Kagels von den 'Darmstädter Ferienkursen` beeinflusstem Stück Anagrama, während Edward Venn (Lancaster) mit Thomas Adès' Freaky, Funky Ravedas Gebiet der elektronischen Tanzmusik (EDM) beschritt.

Pop

Im Vergleich zu den vergangenen Kongressen, die den Bereich der Popmusik nur vereinzelt streiften, kam ihm diesmal die Hauptrolle in immerhin acht Vorträgen zu. Grundsatzfragen zu Analyse und Interpretation dieser Musikrichtung thematisierten Philippe Gonin (Bourgogne) und Volkmar Kramarz (Bonn). Auch Dai Griffiths (Oxford) sprach die Methodologie der Analyse an - nunmehr fokussiert auf den Text im Sänger-SongwriterRepertoire. Raphael D. Thöne (Düsseldorf/Boston) erörterte Malcolm Arnolds The Three Musketeers. In Heinrich W. Schwabs (Kopenhagen) Vortrag zu Scott Joplins Magnetic Rag, war erneut das Thema `Aufführung` zentral. Interpretationsvergleiche standen im Zentrum der Referate von Enrico Bianchi (Perugia), der Bearbeitungen von Antonio Carlos Jobims Aguas de Marco vorstellte, und Daniel DiPaolo (Columbia), der Brad Mehldaus frühe Musik untersuchte. 


\section{Neue Forschungsrichtungen}

\section{Performance Studies}

Was zuvor bereits in einzelnen Referaten verschiedener Sektionen diskutiert wurde, fand in den »Performance Studies« eine abschließende Fokussierung: der Zusammenhang zwischen musikalischer Analyse und Aufführung. Methodisch geschah dies überwiegend anhand von Interpretationsvergleichen, so etwa in den Vorträgen Miriam Quicks (London) über Weberns Variationen op. 27 und Mario Vogts (Düsseldorf/Essen) zur musikalischen Zeitgestaltung in Beethovens letzter Klaviersonate. Auch ein Vergleich verschiedener Aufnahmen desselben Interpreten kann erhellend sein, wie Christine Esclapez (Aix-Marseille) in Bezug auf Boulez' Einspielungen von Igor Strawinskys Le Sacre du printempszeigte. Interessant gestaltete sich auch Hans Peter Reutters (Hamburg) Analyse einer Interpretation durch den Komponisten selbst, im Fall der Sinfonie in C durch Strawinsky.

\section{Ethnologie}

In der Sektion »Ethnologie«, ein im Kontext bisheriger GMTH-Kongresse kaum beachtetes Gebiet, lag der Schwerpunkt auffällig bei französischsprachigen Forschungsbeiträgen. Die einzige Ausnahme bildete der Vortrag Hubertus Dreyers (Tokyo), der sich mit dem »Wort-Ton-Verhältnis in japanischer Jiuta-Musik« auseinandersetzte.

\section{Kongresse im Kongress}

\section{Formenlehre}

Ein Höhepunkt des Kongresses war zweifellos die Plenums-Veranstaltung mit international anerkannten Größen auf dem Gebiet der Formenlehre. Pieter Bergé gab in seiner Eigenschaft als Chair zunächst einen Überblick über den aktuellen Stand der Diskussion, worauf James Hepokoski (Yale) vor dem Hintergrund der Sonaten-Theorie ein »dialogisches Formmodell« generierte, bei dem das individuelle Werk im Verhältnis zu den historischen Standards untersucht wird. James Webster (Ithaca) machte auf das wieder aufkeimende Interesse am Formbegriff, speziell in den USA, aufmerksam und hob zum einen die Interaktion verschiedener Parameter, zum anderen das Theorie-Praxis-Verhältnis als noch ausstehende Forschungsgegenstände hervor. William Caplin (McGill) schließlich differenzierte sein Konzept der `Formfunktionen` in Abgrenzung zu Formtypen mit Hilfe verschiedener hierarchischer Schichten. Abgerundet wurde die Veranstaltung durch eine lebhafte Diskussion am Roundtable.

\section{Symposium "Stimme»}

Richard Klein eröffnete das von ihm geleitete Symposium mit einer Reflexion »über Schwierigkeiten des Verstehens von Stimme«. Dabei thematisierte er psychologische 
wie ontologische Fragen und kam zu dem Schluss, die Stimme als Interpretationswerkzeug in der klassischen Gesangskultur müsse unterschieden werden von der Stimme im Blues- und Rockgesang. Hieran anknüpfend nahm Sonja Dierks (Freiburg) einen Vergleich der Gesangsleistungen Maria Callas' und Björks vor, während Christian Bielefeldt (Zürich) auf Besonderheiten der "Stimme im Jazz-Age« hinwies. Auf neue tonmeisterliche Möglichkeiten bei der Einspielung von Opernstimmen machte Johanna Dombois (Bonn) aufmerksam. Petra Gehring (Darmstadt) fragte nach dem Zusammenspiel von stimme und ‘Lüger. Die »Paradoxie« der im Werk abwesenden, gleichwohl als sfehlend s mitgedachten Stimme in Luigi Nonos Streichquartett Fragmente - Stille, An Diotima entfaltete Ingrid Allwardt (Witten/Herdecke). Wolfgang Fuhrmann (Berlin) erörterte den „Wandel der Stimmästhetik zwischen Spätmittelalter und Früher Neuzeit» und Heinrich Detering (Göttingen) beendete das Symposium mit einer Analyse von »Dylans Stimme im Radio .

\section{Symposium »Zahl und Struktur in Josquins Werk»}

Eingeleitet durch seinen Chair Christian Berger, begann das Symposium mit Helmut Satzingers (Wien) Beobachtungen zur »Gematrie in einem koptischen Zaubertext«. Hans W. Hubert (Freiburg) gab einen Abriss über die »mathematische Struktur italienischer Baukunst zur Zeit von Josquin«. Um den theoretisch-philosophischen Hintergrund ging es Anja Heilmann (Jena), die sich mit »Boethius' anagogischer Musiktheorie« beschäftigte. Orm Finnendahl (Freiburg) machte auf Zahlenbezüge in einer Josquin-Motette aufmerksam. Helmutz G. Walther (Jena) und Guido Heidloff (Hannover) thematisierten jeweils die Voraussetzungen des Zahlenkomponierens. Walther hatte dabei mit den »Grundlagen des Zählens in der quadrivialen Ausbildung der Universität Paris« in erster Linie den Bildungshintergrund im Blick, wohingegen Heidloff die Frage nach Relevanz und Implikationen von Zahlen als kompositionskonstitutives Merkmal aufwarf. Von besonderem methodischen Interesse war Rainer Bayreuthers (Göttingen) »wahrscheinlichkeitstheoretische Untersuchung zahlhafter Analysen«. Regina Randhofer (Leipzig) thematisierte frühe Bibelübersetzungen, was Peter Walter (Freiburg) um eine »Übersetzungsgeschichte der lateinischen Psalmen« ergänzte. Den Abschluss bildete Christian Bergers und Gösta Neuwirths (Berlin) gemeinsamer Vortrag zu Josquins Miserere.

\section{Workshops und Konzerte}

Auflockernd wirkte der von Markus Jans und Dominique Muller (beide Basel) geleitete Workshop mit dem provokanten Titel »Orgasmus oder Tod«. Unterstützt durch die sängerische Mitwirkung einiger Studenten der Musikhochschule Freiburg wurde die vielfältige Musikalisierung der Worte smorire und >dolce morire` in Madrigalen des 16. und 17. Jahrhunderts anschaulich. Fließend war so die Grenze zu den drei abendlichen Konzerten, die das musikalische Rahmenprogramm des Kongresses bildeten. Dabei griffen Hans-Jörg Mammel (Tenor) und Michael Behringer (Fortepiano) mit der ersten Darbietung den Titel der Sektion »Musik und Text« auf und überraschten das Publikum mit einer Zusammenstellung aus Schuberts Winterreise, begleitet am Hammerklavier. »Zum 
Begriff der Improvisation im 18. Jahrhundert« lautete der Titel des zweiten Konzerts, das von Robert Hill, Michael Behringer (beide Cembalo und Fortepiano) und Gottfried von der Goltz (Violine) gestaltet wurde. Teodoro Anzellotti schließlich widmete sich am letzten Abend eindrucksvoll der Interpretation Neuer Musik am Akkordeon.

\section{Resumee}

Trotz seiner großen thematischen Bandbreite spiegelte der Freiburger Kongress deutlich einige Entwicklungstendenzen der Musiktheorie in den vergangenen Jahren. Ruft man sich die Fragestellung des mittlerweile sechs Jahre zurückliegenden GMTH-Gründungskongresses ins Gedächtnis, wie Musiktheorie sich "zwischen Historie und Systematik» verorten lasse, so wird deutlich, dass die Konjunktur historisch orientierter Ansätze seither noch zugenommen hat. Eher selten begegneten Beiträge mit ausgesprochen systematischem und theoriebildendem Anliegen. Dies mag zunächst vor dem Hintergrund verständlich erscheinen, dass sich in der Vergangenheit sSystematikı zu oft mit einer ahistorischen >Dogmatikı verband. Doch stellt sich die Frage, inwiefern die vielen individuellen Werkanalysen den musiktheoretischen Diskurs voranbringen können, sofern sie den systematischen Darstellungen nur entgegengestellt sind. Hier erschienen eine stärkere Integration systematischer Ansätze und deren historische Ausdifferenzierung wünschenswert.

Wie die Gewichte zukünftig gelagert sind, wird nicht zuletzt durch die Auseinandersetzung mit der nordamerikanischen Musiktheorie bestimmt werden. Deren maßgebliche Rolle war in Freiburg unübersehbar. Vielleicht am deutlichsten warf das Panel zur Sektion »Formenlehre« mit der, abgesehen vom Gastgeber, rein amerikanischen Besetzung die Frage nach der Eigenständigkeit und Relevanz europäischer Forschungstraditionen auf.

Eine Vielzahl von Beiträgen spiegelte die Tendenz zu verstärkter Interdisziplinarität zwischen der Musiktheorie und den verwandten Forschungsgebieten insbesondere der historischen und systematischen Musikwissenschaft. Einen noch deutlicheren Schritt in diese Richtung darf man vom nächsten Jahreskongress der GMTH erwarten, der vom 9. bis 12. Oktober in Graz stattfinden wird. Unter dem Motto »Musiktheorie als interdisziplinäres Fach« sollen Anknüpfungspunkte zu den angrenzenden Bereichen Musikgeschichte, Musikästhetik und Musikethnologie sowie zur Praxis musikalischer Interpretation und zur kompositorischen Praxis der Gegenwart aufgedeckt werden.

Verena Weidner 